\title{
A Network Coding-Aware Directional Broadcast Routing Scheme for VANET
}

\author{
Normaliza Omar, Naimah Yaakob, Rohani S.M. Farook, Zulkifli Husin, Aini Syuhada Md Zain, Mohamed Elshaikh, and \\ Aznor Hanah Abdul Halim
}

School of Computer \& Communication Engineering, Universiti Malaysia Perlis, Malaysia

\begin{abstract}
Recent technological advancements in wireless communications are fundamentally changing the manner by which devices communicates with one another. Modern wireless devices build networks on their own and aid each other in passing information to any device in the network. The aim of this research is to design and develop a robust routing protocol using network coding technique for Vehicular ad hoc networks (VANET). The purpose of the protocol is to disseminate vehicular traffic to the vehicles approaching the problem area, rather than those leaving the problem area. The designed protocol during the research will be more robust, reliable, and efficient with topology change. In this research, network coding and geographical routing models will be used to develop network coding based VANET routing protocol for alerting vehicles approaching the accident area and emergency message dissemination. The network coding model assumed will serve to increase throughput, in this sense reducing packet loss which will provide a robust broadcast routing protocol.
\end{abstract}

\section{Introduction}

Nowadays, the rapid evolution and cost reduction experienced by wireless communication technologies during the last decade have made them suitable for a wide spectrum of applications. There are a huge number of vehicles travelling along highways and streets around the world and millions of car accidents occur in the world every year. Millions of people injuries and death incidences via automobile accidents each year. Therefore, there is a growing demand for real-time collision avoidance and warning technology to improve driving safety and traffic condition. Due to rapid advancement of technology, vehicles will be comprised with embedded computers, route planner, GSM/GPRS/WCDMA cellular networks, Global Positioning System (GPS), access to road-side wireless sensors devices and WiFi access point [1]. VANETs consist of smart vehicles on the road and provide useful communication services among arbitrary-formed collections of vehicles that are geo-located and with road side infrastructure. Envisioned applications are ranged from critical safety services and advanced driver assistance systems to traffic management and infotainment applications for intelligent transport applications, and comfort applications [2]. For safety applications to work, it is significant that safety message be reliably delivered to the destination vehicle in a timely manner. Thus a number of studies have been performed in designing and developing a reliable routing protocols in VANET [3][4][5]. Whereas, in VANET, a single hop transmission is usually unable to cover all the intended receivers due to limited radio range across a larger distance. Therefore, multi-hop broadcast protocol is necessary to establish a communication between vehicles. As a result, it is necessary to develop reliable and robust routing protocol in VANETs.

The implementation of message dissemination is one of the main challenges of VANET. In VANET network topology changes more rapidly, and then causes frequent network fragmentation (topology hole between platoon vehicles), frequent route breakage, and packet loss. Thus, the routing protocols have been designed for Mobile Ad Hoc Network (MANET) is not applicable on VANET. There are several simulation based studies have been done to investigate the performance of routing protocols between VANET and MANET, in variety traffic scenarios [6][7][8][9][10].

The broadcast routing protocol is used frequently in VANET, such as data sharing and traffic dissemination, thus many researchers have designed and developed suitable broadcast routing protocols for VANET [4][5] [11][12][13]. There are several researches have been executed to study data sharing and establishing a distributed view of the VANET [14][15]. Recent researches have investigated the design of realistic mobility model [16]. Security problems in VANETs are discussed in [17][18][19][20][21][22]. Whereas, this research will fully concentrate on the broadcast routing using network coding. Network coding has recently emerged as a crucial solution of broadcast scenarios in ad hoc networks, and network coding was shown to reliably handle such type of networks and to profoundly enhance the performance. Researchers have investigated network coding in many areas of wireless networking $[23][24]$.

\subsection{High Mobility and Large Obstacle Environment in Cities}

Analyses of traditional routing protocols for mobile ad hoc networks (MANETs) demonstrated that their performance is poor in VANETs [13][25]. The main problem with these protocols (e.g.., AODV [26], DSR [27], etc) in VANETs environments is their route instability in high mobility and large obstacle environments, like cities. The traditional node-centric view of the routes (i.e., an established route is a fixed succession of nodes between the source and destination)

Corresponding author: normaliza@unimap.edu.my 
leads to frequent broken routes in the presence of VANETs' high mobility, as illustrated in Figure 1. Consequently, many packets are dropped and the overhead due to route repairs or failure notifications increases significantly, leading to low delivery ratios and high transmission delays.

An alternative approach is offered by geographical routing protocols (e.g., GFG [28], GOAFR [29], GSR [25], GPSR [30]) which decouple forwarding from the nodes identity; they do not establish routes, but use the position of the destination and the position of the neighbor nodes to forward data. Unlike node-centric routing, geographical routing has the advantage that any node ensuring progress toward the destination can be used for forwarding. For instance, in Figure 1, geographical forwarding could use node N2 instead of $\mathrm{N} 1$ to forward data to D. Route (S, N1, D) established at time $t$ breaks at time $t+\Delta t$ when $N 1$ moves out of the transmission range of $\mathrm{S}$. Despite better path stability, geographical forwarding does not perform well in citybased VANETs either [25][31]. Its problem is that many times it cannot find a next hop (i.e., a node closer to the destination than the current node).
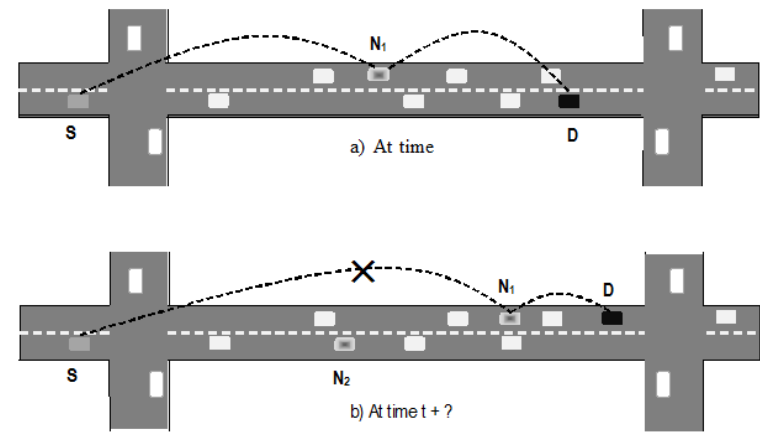

Fig. 1: Routes established as fixed successions of nodes break frequently in highly mobile VANETs.

For example, as shown in Figure 2, it could take road paths that do not lead to destination. Instead of forwarding data on the dotted path, geographical routing sends data to $\mathrm{N} 1$ and $\mathrm{N} 2$, following the shortest geographical path from $\mathrm{S}$ to $\mathrm{D}$ on a dead end road. The recovery strategies proposed in literature are often based on planar graph traversals, which were shown not to be as effective in VANETs due to radio obstacles, high node mobility, and the fact that vehicle positions are constrained on roads rather than being uniformly distributed across a region [25].

A number of road-based routing protocols $[10][13][25][30][32]$ have been designed to address this issue. For example, Geographical Source Routing (GSR) [25] used static street map and location information about each node to avoid GPSR [30] problems. A static street map provides the global knowledge of the city topology to GSR algorithm. The Connectivity-aware Routing Protocol (CAR) [13] is another position-based routing protocol for inter-vehicle communication in a city and/or highway environment. In CAR, the routes are found to destination by maintaining a cache of successful routes between various source and destination pairs. In another attempt, a position-based routing scheme called A-STAR [32] has been proposed to handle disconnection problem due to high rise buildings and trees in city environment. A-STAR [32] also used the static street map to compute the sequence of junctions (anchors) to route messages around radio obstacles. Similarly, a reactive location based routing algorithm is proposed [10] that uses cluster-based flooding technique called LORA_CBF. In LORA_CBF, each node either in any place of cluster can be a cluster, a gateway or an intermediate node. Each cluster must have exactly one cluster-head and if a node is connected to more than one cluster is called a gateway. However, many of these approaches fail to factor in the vehicular traffic flow by using the shortest road path between source and destination.

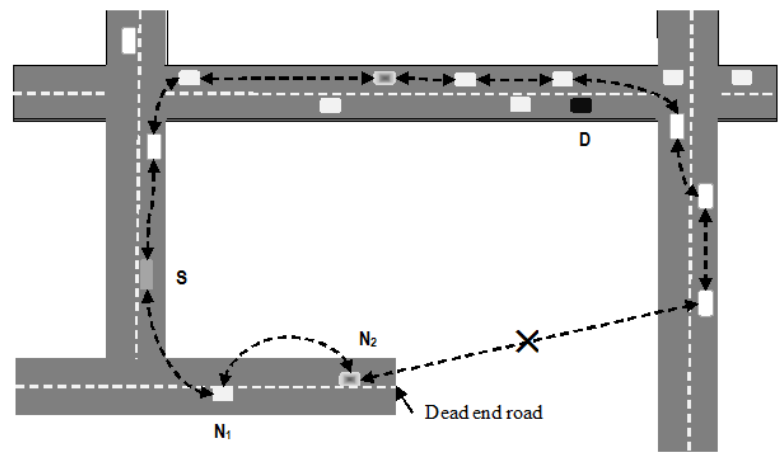

Fig. 2: Geographical routing can route packets toward dead ends causing unnecessary traffic overhead in the network and longer delays for packets

\subsection{High Mobility Environment on Highways/Rural Areas}

Every year traffic accidents are increasing on highways due to automobile crashes. It causes billions of cost in damaged property, nonfatal injuries, and deaths. While different factors contribute to vehicle crashes, such as vehicle mechanical problems, bad weather, and driver behavior. Recently, due to dramatic increase in wireless communication technologies, inter-vehicle networks provide potential solution to control high accident rates on highways.

The efficient VANET routing protocols can play an important role to take in consideration the specifics of highways, relative car movements and application constraints. The main requirement of routing protocols is to achieve minimal communication time with minimum consumption of network resources. Various routing protocols have been developed for Ad-hoc wireless networks, but they cannot be applied directly to intervehicle communication since the characteristics of vehicles movement, information movement and relative speed of mobile nodes are different from that of an Adhoc wireless network. The inability of drivers to react in time to emergency situations often creates a potential for chain collisions, in which an initial collision between two vehicles is followed by a series of collisions involving the following vehicles. The existing MANET 
routing protocols cannot be used in VANET because of highly dynamic topology, frequently disconnection problems, less visibility on slopes, big curves and sharp edges etc (as shown in Figure 3).

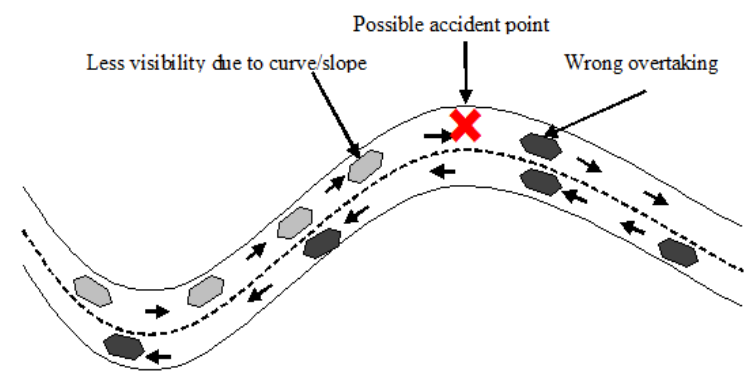

Fig. 3: Rural area scenario on less visibility due to curve/slope

Recently, various VANET routing protocols [11][33] [34][35] have been proposed to handle uncertain situations on highways. An emergency broadcast protocol called BROADCOMM [11] is proposed which is based on a hierarchical structure for a highway network. In BROADCOMM, the highway is divided into virtual cells, which moves as the vehicles move. This protocol outperforms similar flooding based routing protocols in the message broadcasting delay and routing overhead. However, it is very simple and only works with simple highway networks. In [33], authors proposed a cache and distance aware neighborhood selection schemes to deal with the situation of high velocities as compared to regular geocast protocols. A small cache is added within the Zone of Relevance (ZOR) to the routing layer that holds those packets that a node cannot forward instantly due to a local minimum. In [36] also proposed a special geocast routing called abiding geocast. It is a time stable geocast delivered to all nodes that are inside a destination region within a certain period of time. However, the delivery success ratio of these approaches with local message storage is lower in some cases.

\subsection{Vehicular Ad hoc Network (VANET)}

VANET is a special class of mobile ad hoc network (MANET) with their own unique characteristics. Like MANETs [26][27][37], VANETs also do not require any fix infrastructure. However, permanent network nodes can be used in the form of roadside sensor devices. These roadside devices open up a wide variety of services for vehicular networks, such as acting as a drop point for messages on sparsely populated roads, serving up geographically-relevant data, or serving as a gateway to the Internet. VANET has a distinct high speed controlled mobility patterns than other kinds of ad hoc networks (MANETs). It consists of fast moving mobile nodes and large obstacles (e.g., buildings, trees, etc) on highways and urban areas. Speeds of vehicles moving in the same direction are generally similar, and they can therefore remain in radio contact with one another for much longer periods of time than with vehicles moving in the opposite direction.
Because of the unique characteristics of VANETs, the MANETs routing protocols cannot be directly used in VANET efficiently. Several researchers are trying to develop efficient routing protocols that are suitable for VANETs. There are 3 potential areas of research are under investigation in VANETs which includes efficient routing protocols, security, and fairness of bandwidth. However, the routing protocols have only been discussed to study the effect of traffic patterns and congestion in VANETs.

\subsection{Broadcast Routing Protocols}

In this section, broadcasting routing methods in VANETs have been discussed. Broadcasting is a frequently used routing method for sharing traffic information, weather, emergency, road condition, advertisements etc in VANETs. The primary objective of this routing method is to distribute information from a source vehicle to many unknown destinations vehicles. In unicast routing protocols broadcasting method is used to find an efficient route to the destination, Figure 4 and Figure 5 present the difference between unicast and broadcast routing protocols in VANETs. Because of the multi-hop nature of vehicular networks, flooding is a fundamental mechanism to implement the multi-hop broadcasting. Flooding guarantees the message will eventually reach to all nodes in the network. Flooding works well for small number of nodes in the network. However, if number of nodes increase, the bandwidth requested for one broadcast message transmission can also increase exponentially. As each node receives and broadcasts the message almost at the same time, this causes contentions and collisions, broadcast storms and high bandwidth consumption. Flooding may have a very significant overhead and selective forwarding can be used to avoid network congestion.

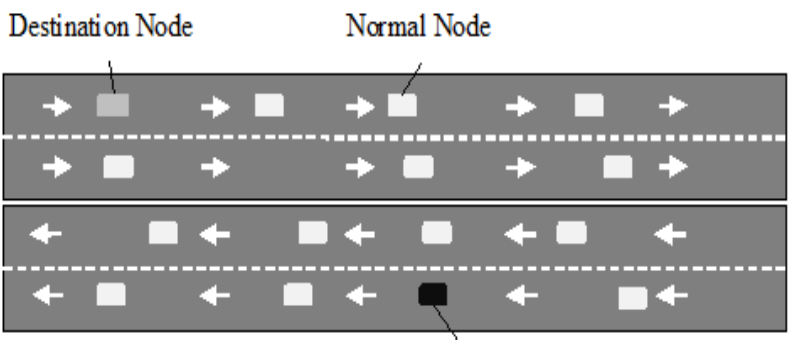

Source Node

Fig. 4: Unicast routing mechanism in VANETs

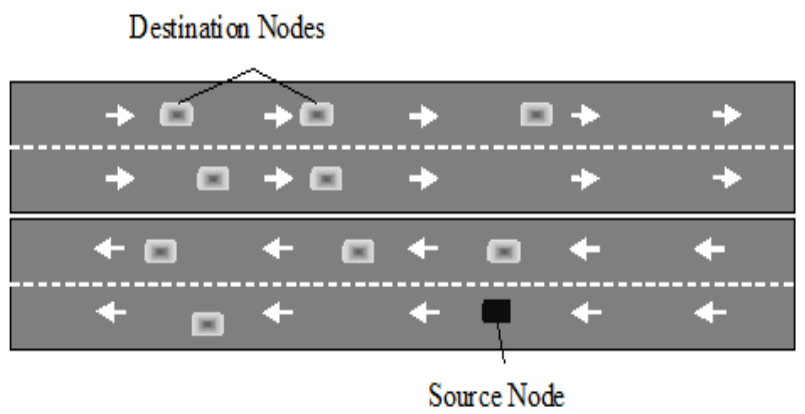

Fig. 5: Broadcast routing mechanism in VANETs 


\subsection{Simulation Setup}

In simulation setup, first, different areas (e.g., $1000 \mathrm{~m} \times 1000 \mathrm{~m}, 1500 \mathrm{~m} \times 1500 \mathrm{~m}$, and $2000 \mathrm{~m} \times 2000 \mathrm{~m}$ ) extracted from the TIGER/Line database of the US Census Bureau [38] will be used to form a grid layout of the road segments. Second, simulation of Urban Mobility (SUMO) [39] will be used to generate the movements of the vehicle nodes. SUMO is an opensource, microscopic, space-continuous and time discrete vehicular traffic generator package. SUMO uses a collision-free car-following model to determine the speeds and positions of the vehicles. The map extracted from the Tiger/Line database as well as specifications about the speed limits and number of lanes of each road segment on the map will be entered into SUMO. The traffic light operated intersections as well as priority intersections (less that one-fifth of the intersections are regulated using traffic lights) will be specified. The first 2000 seconds of SUMO output will be discarded to obtain more accurate node movements. The output from SUMO is converted into input files for the movement of nodes in the NS-2 simulator. The experiments in networks will run with different node densities: the 350 nodes scenario represents relatively dense networks, 250 nodes scenario for medium density networks and 150 nodes for sparse networks.

\subsection{Assumption \& Limitations}

In simulation scenarios, different areas extracted from TIGER/Line database of the US Census Bureau [38] will be used to forms a grid layout with different road segments. Bidirectional traffic will be set on each side of road with two lanes in each direction. In this scenario obstacles are not included in order to evaluate the performance of protocols under increased network congestion. This way, a small increase in data sending rate will provide a noticeable increase in the level of contention in the network. The following points are assumed in this research:

Some data in simulation (e.g., obstacles) is not strictly real-time, there are no real-time constraints on the data, thus in some sense, the data is delay-tolerant.

The number of lanes is not an important and effective parameter in analysis. In fact, the existence of several lanes intensifies the assumption of independent mobility patterns in sparse situations, because the vehicles can overtake each other by simple lane transitions without any hindering effect and any need to speed change. However, the most important factors in analysis are the distribution of the speeds for the vehicles at the arrival instants and their mobility patterns along the street. These factors strongly affect the number of vehicles at the street in the steady state.

- Storage of data is not constrained: VANETs are typified by no power constraints and no data storage limitation.

- Communication between vehicles is over a low data rate connection: While this constraint depends on the radio technology used. Currently, 802.11x devices will offer good-put of the order of a few hundred Kbps.

- Push model: Data is being continually "pushed" by the access points to the nodes in the transmission range.

- Multi-hop delivery: It is infeasible to transmit data to more than 2-3 hops.

\subsection{Expected Results}

Improving the safety of the drivers and passengers by wirelessly exchanging information between vehicles represents a major driving force for the design of vehicular networks. This research proposes the mobility model and a dynamic, cooperative and opportunistic road-based VANET routing mechanism that can cope with heterogeneous vehicles connections. In this research, we are expecting the following results to handle distinguish characteristics of VANETs:

- VANET Routing Protocols for Cities and Highways:

This research is expecting a class of VANET routing protocols for city-based environments that take advantage of the roads layouts to improve the performance of routing in VANETs. The protocols will use real-time vehicular traffic information to create road-based paths between end-points. To improve the end-to-end performance under high contention, it will also be proposed a distributed next hop self-election mechanism for geographical forwarding.

- Realistic Vehicular Environments:

The existing VANET routing protocols are instable due to high mobility and large obstacle environments that leads to frequent broken routes. So, many data packets are dropped and the overhead due to route repairs or failure notifications increases significantly, leading to low delivery ratios and high transmission delays. Therefore, it is expected that proposed technique will outperform the existing approaches in terms of average delivery ratio, average delay, average path length, and overhead. The propose protocols forward data along the streets, not across the streets, and take into account the real traffic on the roads, they perform well in realistic vehicular environments in which buildings and other road characteristics such as dead end streets are present. Such results will show that distributed applications that generate a moderate amount of traffic can be successfully implemented in VANETs.

- Heterogeneous Vehicle Connections:

Heterogenity is important because there may be many vehicles are trying to communicate with each other and exchanging useful information. Therefore, it is expected that proposed vehicular network system will be able to handle such situations. 


\section{CONCLUSION}

It is a big challenge to handle the distinguish characteristics of VANETs such as highly dynamic topology, frequently disconnected networks, various communications environments, and hard delay constraints. The aim of this research is to focus on these VANETs special characteristics. In particular, first mobility model based on car-following and lanechanging Gipps model will be developed to design network protocols. Then, reactive and proactive routing algorithm will be designed to discover routes on-demand basis and generate periodical connectivity to store route information in terms of graphs respectively. Finally, a protocol will be designed to cope with the network delay, loss, and mobility while meeting the application constraints.

\section{REFERENCES}

[1] Chao, H. C., and Zeadally, S. (Eds.). Mobility protocols for ITS/VANET. Elsevier, Computer Communications 31 (2008) 2765-2766(2008).

[2] Sichitiu, M., and Kihl, M., Inter-vehicle communication systems: a survey, IEEE Communications Surveys \& Tutorials, Vol. 10, Issue 2, pp. 88-105, (2008).

[3] Ros, F., Ruiz, P., and Stojmenovic, I., Reliable and Efficient Broadcasting in Vehicular Ad Hoc Networks. Vehicular Technology Conference IEEE, Barcelona, 26-29 April 2009, pp. 1 - 5 (2009).

[4] Lai, P., Wang, X., Lu, N., and Liu, F., A Reliable Broadcast Routing Scheme Based on Mobility Prediction for VANET. Intelligent Vehicles Symposium IEEE, Xi'an, China, 3-5 June 2009, pp. 1083 - 1087 (2009).

[5] Yadumurthy, R., Chimalakonda, A., Sadashivaaiah, M.,Makanaboyina, R., Reliable MAC Broadcast Protocol in Directional and omni-Directional Transmissions for Vehicular Ad hoc Networks. In Proceedings of the 2nd ACM international workshop on Vehicular ad hoc network, Germany, 2005, pp. 10 - 19 (2005).

[6] Namboodiri, V., Agarwal, M., and Gao, L., “A study on the feasibility of mobile gateways for vehicular ad-hoc networks.," in Proceedings of the First International Workshop on Vehicular Ad Hoc Networks, pp. 66-75, (2004).

[7] Liu, G., Lee, B.-S., Seet, B.-C., Foh, C.H., Wong, K.J., and Lee, K.-K., "A routing strategy for metropolis vehicular communications,'” in International Conference on Information Networking (ICOIN), pp. 134-143, (2004).

[8] Fu"ßler H., Mauve M., Hartenstein H., Kasemann M., and Vollmer, D., "Locationbased routing for vehicular ad-hoc networks," ACM SIGMOBILE Mobile Computing and Communications Review (MC2R), vol. 7, no. 1, pp. 47-49, January 2003, (2003).
[9] Wang S.Y., Lin C.C., Hwang Y.W., Tao K.C., and Chou C.L., "A practical routing protocol for vehicle-formed mobile ad hoc networks on the roads," in Proceedings of the 8th IEEE International Conference on Intelligent Transportation Systems, 2005, pp. 161-165, (2005).

[10] Santos, R. A., Edwards A., Edwards R., and Seed L., "Performance evaluation of routing protocols in vehicular ad hoc networks," The International Journal of Ad Hoc and Ubiquitous Computing, vol. 1, no. 1/2, pp. 80-91, (2005).

[11] Durresi, M., Durresi, A., and Barolli, L.,. Emergency broadcast protocol for inter-vehicle communications. In ICPADS '05: Proceedings of the 11th International Conference on Parallel and Distributed Systems-Workshops (ICPADS'05), (2005).

[12] Korkmaz, G., Ekici, E., Özgüner, F., and Özgüner, Ü.,. Urban multi-hop broadcast protocol for inter-vehicle communication systems. In ACM International Workshop on Vehicular Ad Hoc Networks, pp. 76-85, (2004).

[13] V. Naumov and T. Gross, "Connectivity-Aware Routing (CAR) in Vehicular Ad Hoc Networks," In Proc. of the IEEE International Conference on Computer Communications (INFOCOM'07), May, (2007).

[14] M.Y.Sanadidi ,A. Nandan, S. Das, G. Pau, and M. Gerla, , "Co-operative downloading in vehicular ad-hoc wireless networks," in Second Annual Conference on Wireless Ondemand Network Systems and Services (WONS), pp. 32-41, (2005).

[15] Nandan A., Das S., Tewari S., Gerla M., and Klienrock L., "Adtorrent: Delivering location cognizant advertisements to car networks," in The Third International Conference on Wireless On Demand Network Systems and Services(WONS 2006), (2006).

[16] Saha, A.K., and Johnson, D.B.,. Modeling mobility for vehicular ad-hoc networks. In Proceedings of the 1st ACM International Workshop on Vehicular Ad Hoc Networks, VANET'04, ACM, New York, NY, USA, (2004).

[17] Choi, Jaeduck and Jung, Souhwan "A security framework with strong non-repudiation and privacy in VANETs" in 6th IEEE Consumer Communications and Networking Conference, (2009).

[18] Broustis, I., and Faloutsos, M.,. Routing in vehicular networks: feasibility, security, and modeling issues. Tech. Rep. UCR-CS-200605219, Department of Computer Science and Engineering, University of California, Riverside, Oct 2006, (2006).

[19] Eichler, S., Dötzer, F., Schwingenschlögl, C., 
Caro, F.J.F., and Eberspächer, J.,. Secure routing in a vehicular ad hoc network. In: Proceedings of the IEEE 60th Vehicular Technology Conference, VTC2004-Fall, IEEE, (2004).

[20] Fonseca, E., and Festag, A.,. A survey of existing approaches for secure ad hoc routing and their applicability to VANETS. Tech. Rep. NLE-PR-2006-19, NEC Network Laboratories, Mar 2006, (2006).

[21] Golle, P., Greene, D., and Staddon, J.,. Detecting and correcting malicious data in VANETs. In: Proceedings of the 1st ACM International Workshop on Vehicular Ad Hoc Networks, VANET'04, ACM, New York, NY, USA, (2004).

[22] Raya, M., and Hubaux, J.-P.,. The security of vehicular ad hoc networks. In: Proceedings of the 3rd ACM Workshop on Security of Ad Hoc and Sensor Networks, SASN'05, ACM, New York, NY, USA, (2005).

[23] Fragouli, C., WIdmer, J., and LeBoudec, J.,"Efficient broadcasting using network coding" IEEE/ACM Transactions on Networking, (2007).

[24] Katti, S., Rahul, H., Katabi, D., Hu, W., M'edard, M., and Crowcroft. J., "XORs in the Air: Practical Wireless Network Coding" In ACM SIGCOMM, (2006).

[25] Lochert, C., Hartenstein, H., Tian, J., Herrmann, D., Füßler, H., and Mauve, M.,A routing strategy for vehicular ad hoc networks in city environments. In Proceedings of IEEE Intelligent Vehicles Symposium (IV2003), pp. 156-161, June 2003, (2003).

[26] Perkins, C.E., and Royer, E.M., (1999). Ad-hoc on demand distance vector routing. In Proceedings of the 2nd IEEE Workshop on Mobile Computing Systems and Applications, pp. 90-100, Feb. 1999, (1999).

[27] Johnson, D., and Maltz, D.,. Dynamic Source Routing in Ad Hoc Wireless Networks. Mobile Computing, T. Imielinski and H. Korth, Ed., pp. 153-181. Kluwer, (1996).

[28] Bose, P., Morin, P., Stojmenovic, I., and J. Urrutia,. Routing with guaranteed delivery in ad hoc wireless networks. ACM Wireless Networks, vol. 7, no. 6, pp. 609-616, November 2001, (2001).

[29] Kuhn, F., Wattenhofer, R., Zhang, Y., and Zollinger, A., (2003). Geometric ad-hoc routing: Of theory and practice. In Proceedings of the twenty-second annual symposium on Principles of distributed computing, Boston, MA, USA, July 2003, pp. 63-72, (2003).

[30] Karp, B., and Kung, H.T.,. GPSR: Greedy perimeter stateless routing for wireless networks. In Proceedings of the ACM/IEEE International Conference on Mobile Computing and
Networking (MobiCom), (2000).

[31] Li, T., Hazra, S. K., and Seah, W.,. A positionbased routing protocol for metropolitan bus networks. In Proceedings IEEE 61st Vehicular Technology Conference VTC-Spring, Stockholm, Sweden, June 2005, pp. 2315-2319, (2005).

[32] Seet, B.-C., Liu, G., Lee, B.-S., Foh, C.-H., Wong, K.-J., and Lee, K.-K.,. A-STAR: A mobile ad hoc routing strategy for metropolis vehicular communications. In: Proceedings of the Third International IFIP-TC6 Networking Conference, Networking Technologies, Services, and Protocols; Performance of Computer and Communication Networks; Mobile and Wireless Communications, NETWORKING 2004, in: Lecture Notes in Computer Science, vol. 3042/2004, Springer, Berlin, Heidelberg, 2004, pp. 989-999, (2004).

[33] Maihöfer C., and Eberhardt, R.,. Geocast in vehicular environments: Caching and transmission range control for improved efficiency. In Proceedings of IEEE Intelligent Vehicles Symposium (IV), pp. 951-956, (2004).

[34] Sun, M., Feng, W., Lai, T.-H., Yamada, K., Okada, H., and Fujimura, K., GPS-based message broadcasting for inter-vehicle communication. In ICPP '00: Proceedings of the 2000 International Conference on Parallel Processing, (2000).

[35] Ko, Y.-B., and Vaidya, N.H.,. Location-aided routing (LAR) in mobile ad hoc networks. Wireless Networks, vol. 6, no. 4, pp. 307-321, (2000).

[36] Maihöfer, C., Leinmüller, T., and Schoch, E., (2005) Abiding geocast: time-stable geocast for ad hoc networks. In Proceedings of the 2nd ACM international workshop on Vehicular ad hoc networks (VANET '05), pp. 20-29, (2005).

[37] Perkins, C., Belding-Royer, E., and Das, S., (2003). Ad Hoc On demand Distance Vector (AODV) Routing. IETF RFC 3561, July 2003, (2003).

[38] TIGER, U.S.Census Bureau - TIGER/Line 2006 Second http://www.census.gov/geo/www/tiger/ (accessed June 2017), (2008).

[39] SUMO, Centre for Applied Informatics (ZAIK) and the Institute of Transport Research German Aerospace Centre. Sumo - simulation of urban mobility. http://sumo.sourceforge.net/, (2009). 